\title{
Curcumin protects liver inflammation by suppressing expression of inducible nitric oxide synthase in primary cultured rat hepatocytes
}

\author{
Richi Nakatake $^{1 *}$, Hidehiko Hishikawa ${ }^{1}$, Hideyuki Matushima ${ }^{1}$, Yusuke Nakamura $^{1}$, \\ Morihiko Ishizaki $^{1}$, Kosuke Matsui ${ }^{1}$, Masaki Kaibori ${ }^{1}$, Mikio Nishizawa ${ }^{2}$, Tadayoshi \\ Okumura $^{1,3}$, and Masanori Kon ${ }^{1}$
}

${ }^{1}$ Department of Surgery, Kansai Medical University, Hirakata, Osaka, Japan; ${ }^{2}$ Department of Biomedical Sciences, College of Life Sciences; and ${ }^{3}$ Research Organization of Science and Technology, Ritsumeikan University, Kusatsu, Shiga, Japan

Submission Date: May $7^{\text {th }}, 2017$, Acceptance Date September $27^{\text {th }}, 2017$, Publication Date: September $30^{\text {th }}, 2017$,

Citation: Nakatake R., Hishikawa H., Matsuhima H., Nakamura Y., Ishizaki M., Matsui K., Kaibori M., Nishizawa M., Okumura T., Kon M., Curcumin protects liver inflammation by suppressing expression of inducible nitric oxide synthase in primary cultured rat hepatocytes. Functional Foods in Health and Disease 2017; 7(9); 716-734

Running title: Curcumin inhibits hepatic iNOS induction

*Correspondence: Richi Nakatake, M.D. and Ph.D., Department of Surgery, Kansai Medical University, 2-5-1 Shinmachi, Hirakata, Osaka, 573-1010, Japan

\begin{abstract}
Background: Curcumin has beneficial effects on organ metabolism. However, there is little evidence that curcumin affects inflammatory mediators, such as tumor necrosis factor (TNF)- $\alpha$ and nitric oxide (NO). In an inflamed liver, proinflammatory cytokines stimulate liver cells, followed by the induction of inducible NO synthase (iNOS). Excessive NO produced by iNOS is one of the factors in liver injury. Therefore, inhibiting iNOS induction for preventing liver injury is important.
\end{abstract}

Objective: This study aimed to investigate liver protective effects of curcumin by examining interleukin (IL)-1 $\beta$-stimulated hepatocytes. 
Methods: Primary cultured rat hepatocytes were treated with IL-1 $\beta$ in the presence or absence of curcumin. Induction of NO production and iNOS, and the signaling pathway of iNOS were analyzed.

Results: Simultaneous addition of IL-1 $\beta$ and curcumin decreased expression levels of iNOS protein and mRNA, resulting in inhibition of NO production. Curcumin also reduced mRNA expression of TNF- $\alpha$ and IL-6. Curcumin inhibited two essential signaling pathways for iNOS induction, NF- $\mathrm{\kappa B}$ activation and type I IL-1 receptor upregulation. Transfection experiments revealed that curcumin reduced iNOS mRNA levels at the promoter activation and mRNA stabilization steps. Delayed administration of curcumin after IL- $1 \beta$ addition also inhibited iNOS induction.

Conclusions: Curcumin affects induction of inflammatory mediators, such as iNOS and TNF- $\alpha$, in part through the inhibition of NF- $\mathrm{\kappa B}$ activation in hepatocytes. Curcumin may have therapeutic potential for organ injuries, including the liver.

Key words: curcumin, inducible nitric oxide synthase, liver injury, primary cultured hepatocytes, nuclear factor- $\mathrm{\kappa B}$, type I interleukin-1 receptor, tumor necrosis factor- $\alpha$.

\section{INTRODUCTION:}

Attention is being focused on the new academic discipline of anti-aging medicine, which aims to slow age-related pathological changes. One important research subset in this field involves foods and herbs that are known to be particularly effective for maintaining and enhancing health. These foods of interest have been shown to have remarkable pharmacological effects, comparable with those of existing pharmaceutical products. Therefore, substantial research efforts are currently ongoing to determine the active compounds that are responsible for these positive effects.

Curcumin, a diferuloyl-methane derived from the rhizome of Curcuma longa, is the active component of the spice turmeric (Fig. 1) [1]. Turmeric and curcumin have been used for thousands of years in Southeast Asia and India in a variety of medicinal applications [2]. Traditionally, curcumin has been used in Chinese and Indian folk medicine to treat various diseases and health problems, including respiratory conditions, anorexia, rheumatism, liver disease, and cancer. In recent years, some studies have investigated the various biological effects of curcumin [3, 4]. In addition to the reported cytoprotective effect of curcumin, curcumin has an antioxidant effect by removing free radicals and an anti-inflammatory effect by inhibiting activation of nuclear factor kappa B (NF-кB) [4]. Two clinical trials that investigated curcumin concluded that this compound may be useful in preventing heart failure and effective against a range of diseases, including cancer $[4,5]$. 


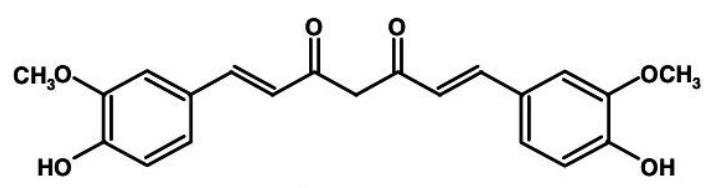

Fig. 1. Structure of curcumin.

In hepatic disorders, inflammatory cells such as macrophages gather around hepatic stellate (Kupffer) cells and discharge a variety of cytokines. During inflammation, proinflammatory cytokines and nitric oxide (NO), which is produced by inducible nitric oxide synthase (iNOS) in Kupffer cells and hepatocytes, play an important role as factors in liver injury [6]. However, NO exerts either detrimental or beneficial effects, depending on the insults and tissues involved.

In animal liver injury models caused by various insults, such as ischemia-reperfusion, partial hepatectomy, and endotoxin shock, we have previously reported that drugs [7-11] demonstrated how liver-protective effects inhibit induction of iNOS and NO production. These drugs also decrease production of various inflammatory mediators, such as tumor necrosis factor (TNF)- $\alpha$, interleukin (IL)-1 $\beta$, IL-6, and cytokine-induced neutrophil chemoattractant (CINC)-1 (human IL8 analogue) [7-11].

We previously reported that in hepatocytes that were isolated by the collagen perfusion method from rats and cultured the proinflammatory cytokine IL-1 $\beta$ stimulated induction of iNOS gene expression in primary cultures of rat hepatocytes [12]. Previous studies on IL-1 $\beta$-stimulated hepatocytes have shown that clinical drugs $[9,13,14]$ as mentioned above also inhibit induction of iNOS and production of NO. Therefore, by using cultured hepatocytes, prevention of iNOS induction and NO production is considered to be one of the indicators of liver protection [15].

In the present study, we examined IL-1 $\beta$-stimulated hepatocytes and investigated the mechanisms underlying liver-protective effects of curcumin that are observed in various animal models. We examined whether curcumin affects induction of iNOS, and if so what mechanisms were involved in the action of curcumin.

\section{MATERIALS AND METHODS:}

Materials. Curcumin (Wako Pure Chemicals, Osaka, Japan) was dissolved in dimethyl sulfoxide (DMSO) at $100 \mathrm{mM}$ and stored at $-20^{\circ} \mathrm{C}$. The final concentration of DMSO in the culture medium was $0.1 \%$ or less. Recombinant human IL-1 $\beta\left(2 \times 10^{7} \mathrm{U} / \mathrm{mg}\right.$ protein $)$ was purchased from MyBioSource (San Diego, CA, USA). Male Wistar rats (200-250 g and 6-7 weeks old) were purchased from Charles River (Tokyo, Japan), kept at $22^{\circ} \mathrm{C}$ under a 12:12 h light:dark cycle, and received food and water ad libitum. All experiments on rats were performed in accordance with the Guidelines for the Care and Use of Laboratory Animals of the National Institutes of Health. The experiments were approved by the Animal Care Committee of Kansai Medical University. 
Primary cultures of hepatocytes. Hepatocytes were isolated from rats by perfusion with collagenase (Wako Pure Chemicals) [16]. Isolated hepatocytes were suspended in a culture medium at $6 \times 10^{5}$ cells $/ \mathrm{mL}$, seeded into $35-\mathrm{mm}$ plastic dishes $(2 \mathrm{~mL} / \mathrm{dish}$; Falcon Plastic, Oxnard, $\mathrm{CA}$, USA), and cultured at $37^{\circ} \mathrm{C}$ in a $\mathrm{CO}_{2}$ incubator under a humidified atmosphere of $5 \% \mathrm{CO}_{2}$ in air. The culture medium was WE supplemented with $10 \%$ newborn calf serum, $10 \mathrm{nM}$ insulin, and $10 \mathrm{nM}$ dexamethasone. After $5 \mathrm{~h}$, the medium was replaced with fresh serum and hormone-free WE, and the cells were cultured overnight before use in experiments. The numbers of cells attached to the dishes were calculated by counting the number of nuclei [17] and using a ratio of $1.37 \pm$ 0.04 nuclei/cell (mean $\pm \mathrm{SE}, \mathrm{n}=7$ experiments).

Treatment of cells with curcumin. On day 1, the cells were washed with fresh serum- and hormone-free WE and incubated with IL-1 $\beta(1 \mathrm{nM})$ in the same medium in the presence or absence of curcumin. The doses of curcumin used are indicated in the appropriate figures and their legends.

Determination of NO production and lactate dehydrogenase (LDH) activity. Culture medium was used for measurements of nitrite (a stable metabolite of NO) to reflect NO production by the Griess method [18]. Culture medium was also used for measurements of LDH activity to reflect cell viability using a commercial kit (Roche Diagnostics, Mannheim, Germany).

Western blot analysis. Total cell lysates were obtained from cultured cells as described previously with minor modifications [13]. Briefly, cells $\left(1 \times 10^{6}\right.$ cells $/ 35 \mathrm{~mm}$ dish $)$ were lysed with sample buffer for sodium dodecyl sulfate-polyacrylamide gel electrophoresis (SDS-PAGE) (final: 125 $\mathrm{mM}$ Tris- $\mathrm{HCl}, \mathrm{pH}$ 6.8; containing 5\% glycerol, 2\% SDS, and 1\% 2-mercaptoethanol), subjected to SDS-PAGE, and electroblotted onto a polyvinylidene difluoride membrane (Bio-Rad, Hercules, CA, USA). Immunostaining was performed using primary antibodies against mouse iNOS (Affinity BioReagents, Golden, CO, USA), human IkB $\alpha$, mouse type I IL-1 receptor (IL-1RI) (Santa Cruz Biotechnology, Santa Cruz, CA, USA), and rat $\beta$-tubulin (internal control; Clone TUB2.1; Sigma Chemical Co., St. Louis, MO, USA). This was followed by visualization with an enhanced chemiluminescence blotting detection reagent (GE Healthcare Biosciences Corp., Piscataway, NJ, USA).

For Akt, total cell lysates prepared from $100-\mathrm{mm}$ dishes $\left(5 \times 10^{6}\right.$ cells/dish $)$ were pre-cleared with protein A (Sigma Chemical Co.) and then mixed with a mouse monoclonal antibody against human Akt1 (Akt5G3; Cell Signaling, Beverly, MA, USA) and protein G-Sepharose (Pharmacia LKB Biotech, Uppsala, Sweden). After incubation overnight at $4^{\circ} \mathrm{C}$, immunocomplexes were centrifuged $(16,000 \times \mathrm{g}$ for $5 \mathrm{~min})$. The beads were washed with solubilizing buffer, dissolved in SDS-PAGE sample buffer, and analyzed by western blotting using rabbit polyclonal antibodies against human Akt and phospho-Akt (Ser473) (Cell Signaling) as primary antibodies. In the case 
of p65, nuclear extracts were immunoprecipitated with an anti-p65 antibody (H286; Santa Cruz Biotechnology). The bands were analyzed by western blotting using an antibody against human NF- $\kappa$ B p65 (BD Transduction Laboratories, Lexington, KY, USA).

Reverse transcriptase-polymerase chain reaction (RT-PCR). Total RNA was extracted from cultured hepatocytes using the guanidinium-phenol-chloroform method [19]. For strand-specific RT-PCR analysis, cDNA was synthesized from total RNA with strand-specific primers, and stepdown PCR was performed using PC708 (Astec, Fukuoka, Japan) as previously described [20] with minor modifications. For iNOS (257 bp), TNF- $\alpha$ (275 bp), CINC-1 (231 bp), IL-6 (286 bp), IL1RI (327 bp), and elongation factor-1 $\alpha$ (EF; internal control) (335 bp) mRNA, an oligo(dT) primer was used for RT and the indicated primers sets were used for PCR (Table 1). For the antisense transcript of iNOS (211 bp), the sense primer (5'-TGCCCCTCCCCCACATTCTCT-3') was used for RT and the indicated primer set was used for PCR (Table 1). These mRNAs and antisense transcript levels were measured by real-time PCR using the Rotor-Gene Q 2plex HRM (Qiagen, Tokyo, Japan). The Rotor-Gene SYBR Green PCR Kit (Qiagen) was included in the reaction mixture, and the following touchdown protocol was applied: 1 cycle at $95^{\circ} \mathrm{C}$ for 5 min and 45 cycles at $95^{\circ} \mathrm{C}$ for $5 \mathrm{~s}$ and $60^{\circ} \mathrm{C}$ for $10 \mathrm{~s}$. The cDNAs for the rat iNOS mRNA and antisense transcript were deposited in the DNA Data Bank of Japan/European Bioinformatics Institute (DDBJ/EMBL)/GenBank under the accession numbers AB250951 and AB250952 respectively.

Electrophoretic mobility shift assay. Nuclear extracts were prepared and an electrophoretic mobility shift assay was performed as previously described [21]. Briefly, nuclear extracts from hepatocytes $(4 \mu \mathrm{g})$ were mixed with $1 \mu \mathrm{g}$ of poly( $\mathrm{dI}-\mathrm{dC})$ and a probe for $20 \mathrm{~min}$ at room temperature (total mixture, $15 \mu \mathrm{l}$ ). To prepare a double-stranded DNA probe, annealed oligonucleotides harboring a $\kappa \mathrm{B}$ site (5'-AGTTGAGGGGACTTTCCCAGGC-3'; only the sense strand is shown) were labelled with $\left[\gamma_{-}{ }^{32} \mathrm{P}\right]$-adenosine- $5^{\prime}$-triphosphate (ATP; DuPont-New England Nuclear Japan, Tokyo, Japan) and T4 polynucleotide kinase (Takara Bio Inc., Shiga, Japan). Samples were resolved on a $4.8 \%$ polyacrylamide gel, followed by drying and autoradiography. Protein concentrations were measured by the method of Bradford [22] with a binding assay kit (Bio-Rad) using bovine serum albumin as a standard.

Transfection and luciferase assay. Transfection of cultured hepatocytes was performed as previously described [23]. Briefly, hepatocytes were cultured at $4 \times 10^{5}$ cells/dish $(35 \times 10 \mathrm{~mm})$ in WE supplemented with serum, dexamethasone, and insulin for $7 \mathrm{~h}$ before being subjected to magnet-assisted transfection (MATra). The reporter plasmid pRiNOS-Luc-SVpA or pRiNOSLuc-3'UTR $(1 \mu \mathrm{g})$ and the CMV promoter-driven $\beta$-galactosidase plasmid pCMV-LacZ (1 ng) as an internal control were mixed with MATra-A reagent ( $1 \mu \mathrm{l}$; IBA GmbH, Göttingen, Germany). 
After incubation for $15 \mathrm{~min}$ on a magnetic plate at room temperature, the medium was replaced with fresh WE containing serum. Cells were cultured overnight, and then treated with IL-1 $\beta$ in the presence or absence of curcumin. Luciferase and $\beta$-galactosidase activities of cell extracts were measured using PicaGene (Wako Pure Chemicals) and Beta-Glo (Promega, Wisconsin, USA) kits respectively.

Statistical analysis. The results shown are representative of three to four independent experiments yielding similar findings. All data are expressed as means \pm SD. Differences were analyzed by the Bonferroni-Dunn test, and a value of $\mathrm{P}<0.05$ was considered to indicate a significant difference.

\begin{tabular}{ll}
\hline Primer name & Nucleotide sequence \\
\hline iNOS F/R & 5' CCAACCTGCAGGTCTTCGATG 3'/5' GTCGATGCACAACTGGGTGAAC 3' \\
as iNOS F/R & 5' CCTTTGCCTCATACTTCCTCAGA 3'/5' ATCTTCATCAAGGAATTATACACGG 3' \\
TNF- $\alpha$ F/R & 5' ' TCCCAACAAGGAGGAGAAGTTCC 3'/5' GGCAGCCTTGTCCCTTGAAGAGA 3' \\
IL-6 F/R & 5' GAGAAAAGAGTTGTGCAATGGCA 3'/5' TGAGTCTTTTATCTCTTGTTTGAAG 3' \\
CINC-1 F/R & 5' GCCAAGCCACAGGGGCGCCCGT 3'/5' ACTTGGGGACACCCTTTAGCATC 3' \\
IL-1RI F/R & 5'-CGAAGACTATCAGTTTTTGGAAC-3'/5'-GTCTTTCCATCTGAAGCTTTTGG-3' \\
EF F/R & 5' TCTGGTTGGAATGGTGACAACATGC 3'/5' CCAGGAAGAGCTTCACTCAAAGCTT 3' \\
\hline
\end{tabular}

Table 1. Primers and nucleotide sequences

iNOS, inducible nitric oxide synthase; as, antisense transcript; TNF- $\alpha$, tumor necrosis factor-alpha; IL-6, interleukin6; CINC-1, cytokine-induced neutrophil chemoattractant-1; IL-1R1, type I IL-1 receptor; EF, elongation factor-1 $\alpha$; $\mathrm{F} / \mathrm{R}$, forward/reverse.

\section{RESULTS:}

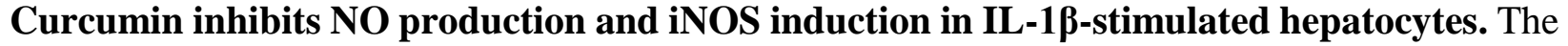
proinflammatory cytokine IL-1 $\beta$ stimulated induction of iNOS, which was followed by production of NO in primary cultured rat hepatocytes. Simultaneous addition of curcumin and IL- $1 \beta$ reduced the levels of nitrite (a stable metabolite of NO) time and dose-dependently in the culture medium (Fig. 2A and 2B). Curcumin showed more than $90 \%$ inhibition at $25 \mu \mathrm{M}$. Curcumin had no cellular cytotoxicity with the various concentrations, as evaluated by the release of LDH into the culture medium (Fig. 3) and Trypan blue exclusion by hepatocytes (data not shown).

Western blotting analysis showed that curcumin dose-dependently reduced the expression of the iNOS protein, showing its maximal effect at $25 \mu \mathrm{M}$ (Fig. 2B). RT-PCR analysis showed that curcumin decreased the expression of iNOS mRNA time-dependently (Fig. 2C). These results suggested that curcumin inhibited the induction of iNOS gene expression at a transcriptional and/or post-transcriptional step. 

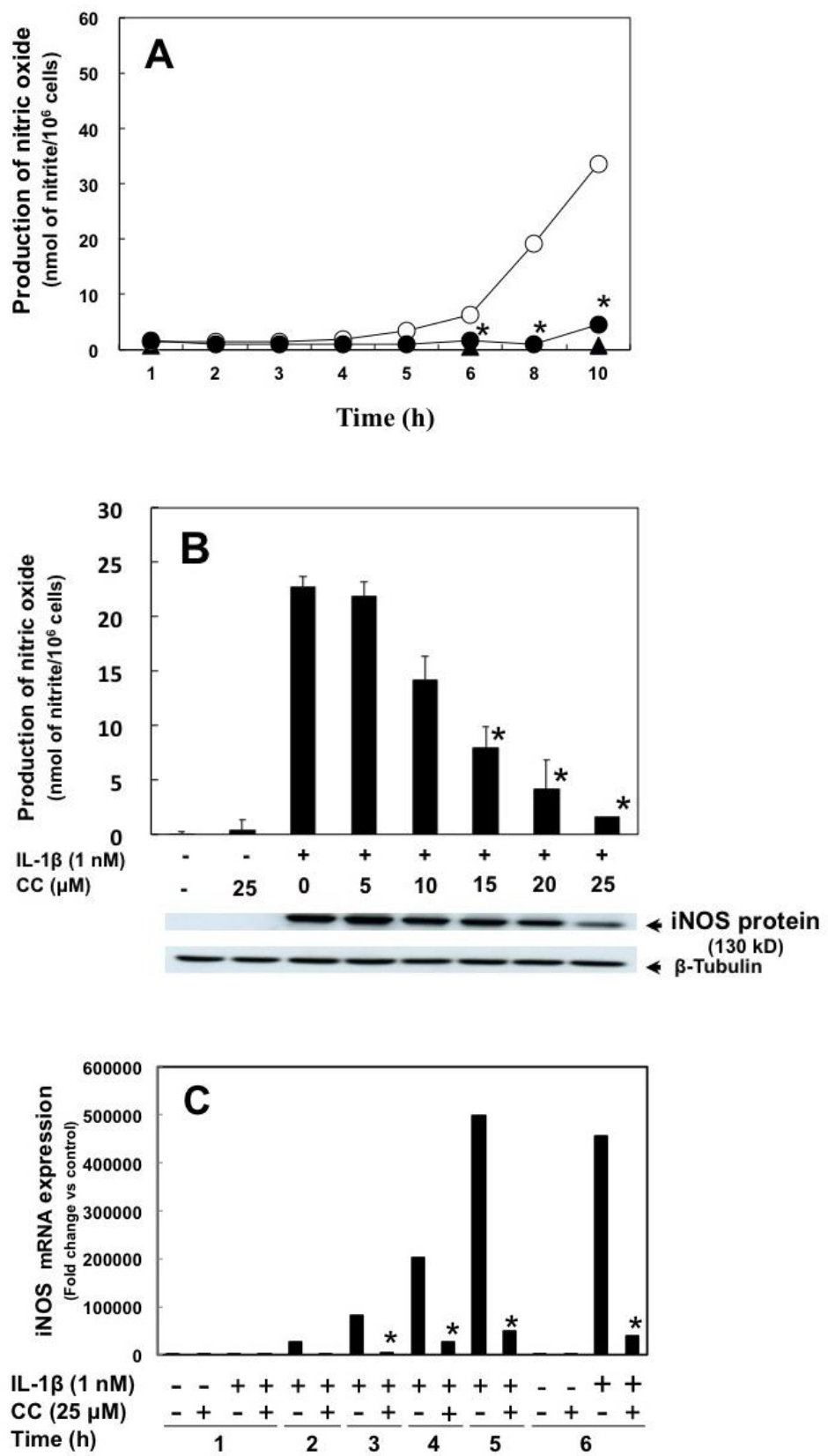

Fig. 2. Effects of curcumin on induction of NO production and iNOS in IL-1 $\beta$-stimulated hepatocytes. Cultured hepatocytes were treated with interleukin (IL)-1 $(1 \mathrm{nM})$ in the presence or absence of curcumin $(5-25 \mu \mathrm{M})$. (A) Effect of curcumin $(25 \mu \mathrm{M})$ treatment for the indicated times on nitric oxide (NO) production (IL-1 $\beta$, open circles; IL-1 $\beta$ + curcumin, filled circles; curcumin, filled triangles; controls [without IL-1 $\beta$ and curcumin], open triangles). (B) Effects of treatment with various doses of curcumin (5-25 $\mu \mathrm{M}$ ) for $8 \mathrm{~h}$ on NO production (upper panel) and inducible nitric oxide synthase (iNOS) protein (medium). Nitrite levels were measured in culture medium. Data are means $\pm \mathrm{SD}$ for $\mathrm{n}=3$ dishes/point; $* \mathrm{P}<0.05$ versus IL- $1 \beta$ alone. In the western blotting panels, cell lysates (20 $\mu \mathrm{g}$ of protein) were subjected to sodium dodecyl sulfate-polyacrylamide gel electrophoresis in a $7.5 \%$ gel, and immunoblotted with an anti-iNOS or anti- $\beta$-tubulin antibody. (C) Effects of curcumin (25 $\mu \mathrm{M})$ treatment for the indicated times on iNOS mRNA expression. Total RNA was analyzed by strand-specific RT-PCR to detect iNOS mRNA, using EF mRNA as an internal control. 


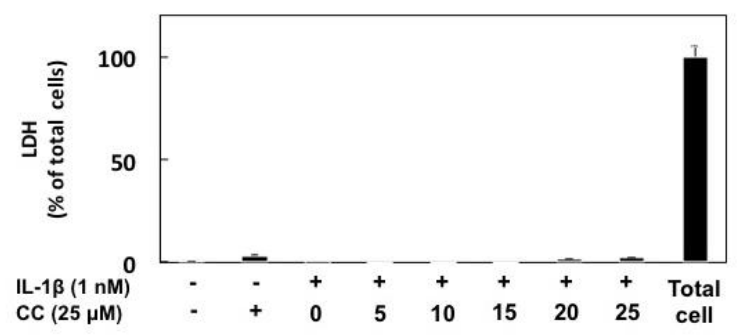

Fig. 3. Effects of curcumin on cellular cytotoxicity. Cells were treated with IL-1 $\beta(1 \mathrm{nM})$ in the presence or absence of curcumin $(5-25 \mu \mathrm{M})$ for $8 \mathrm{~h}$. Lactate dehydrogenase (LDH) activity was measured in the culture medium (data are means $\pm \mathrm{SD}, \mathrm{n}=3$ dishes/point).

Curcumin affects mRNA expression of proinflammatory cytokines. The expression of other mRNAs was examined. IL-1 $\beta$ increased the level of proinflammatory cytokine expression, by increasing TNF- $\alpha$, CINC-1, and IL-6 mRNA levels, and curcumin decreased TNF- $\alpha$, CINC-1, and IL-6 mRNA levels (Fig. 4).
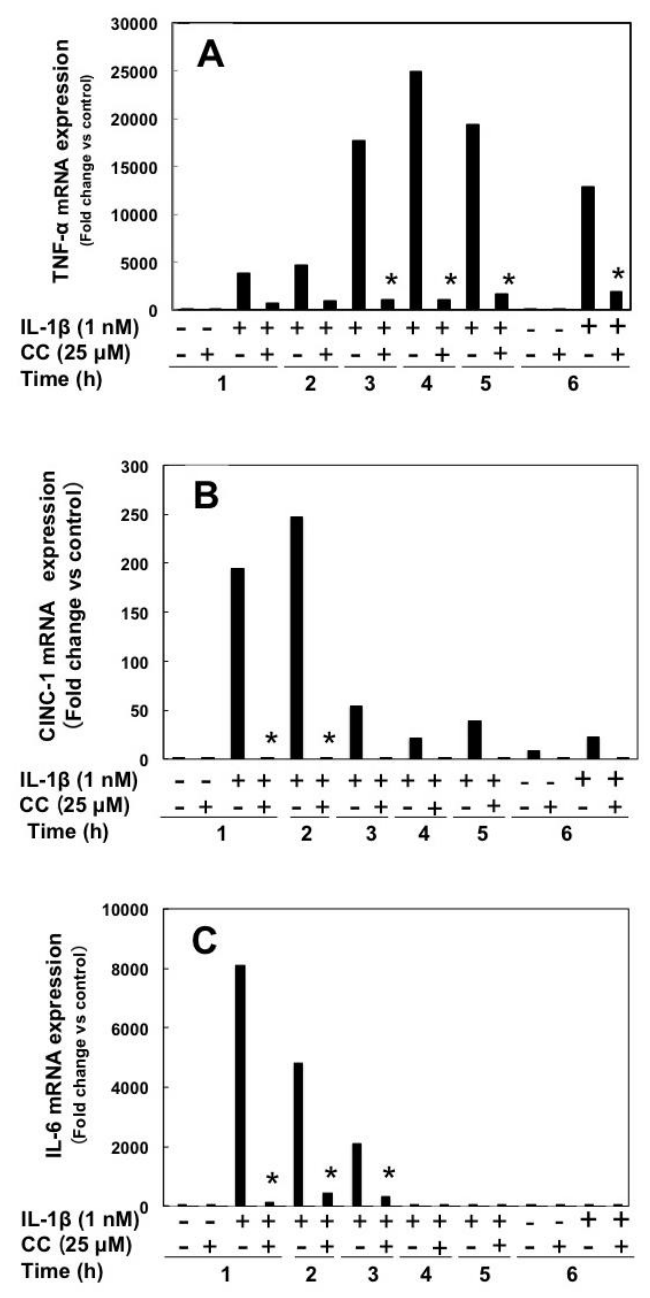

Fig. 4. Effects of curcumin on TNF- $\alpha, \mathrm{CINC}$, and IL-6 mRNA expression. Cells were treated with IL$1 \beta(1 \mathrm{nM})$ in the presence or absence of curcumin $(25 \mu \mathrm{M})$ for the indicated times. Total RNA was analyzed by strandspecific RT-PCR to detect (A) TNF- $\alpha$, (B) CINC-1, and (C) IL-6, using EF mRNA as an internal control. 
Curcumin decreases iNOS mRNA synthesis and stabilization. We examined the mechanisms that are involved in inhibiting iNOS induction. Expression of iNOS mRNA is regulated by iNOS promoter transactivation with transcription factors, such as $\mathrm{NF}-\kappa \mathrm{B}$, and by post-transcriptional modifications, such as mRNA stabilization [24]. Therefore, we carried out transfection experiments with constructs containing firefly luciferase controlled by the iNOS promoter (pRiNOS-Luc-SVpA and pRiNOS-Luc- 3'UTR) (Fig. 5A), which detects iNOS promoter transactivation (mRNA synthesis) and mRNA stabilization respectively. IL-1 $\beta$ increased the luciferase activity of these constructs, and these effects were significantly inhibited by curcumin (Fig. 5B and 5C). Furthermore, iNOS antisense transcript (asRNA) analysis by real-time RT-PCR showed that IL-1 $\beta$ increased the expression of iNOS asRNA in a time-dependent manner, and that curcumin markedly inhibited this effect (Fig. 5D).
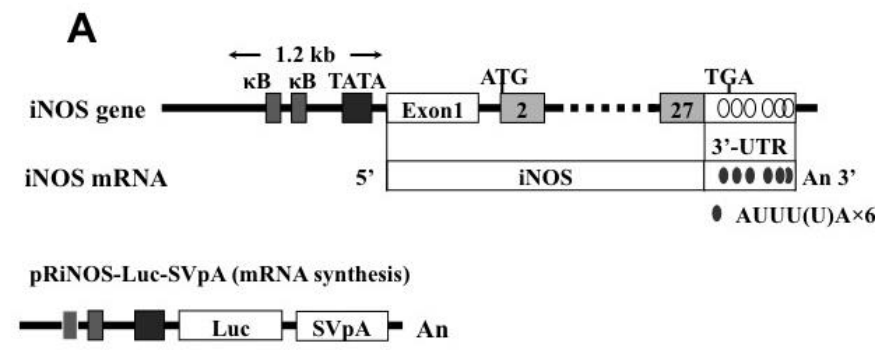

pRiNOS-Luc-3'UTR (mRNA stabilization)
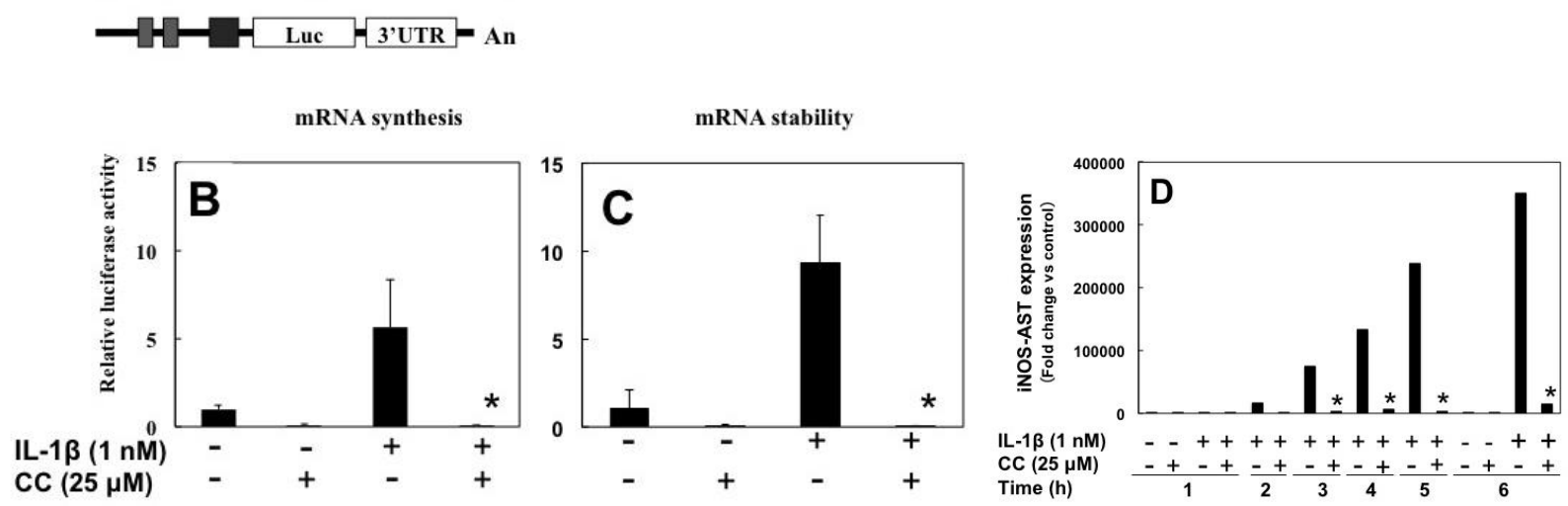

Fig. 5. Effects of curcumin on transactivation of the iNOS promoter and expression of the iNOS gene antisense transcript. (A) Schematic representation of the promoter region of the iNOS gene. Two reporter constructs are shown beneath the iNOS gene and mRNA. The constructs consist of the rat iNOS promoter (1.2 kb), a luciferase gene, and the SV40 poly(A) region (pRiNOS-Luc-SVpA) or iNOS 3'-UTR (pRiNOS-Luc-3'UTR). “An” indicates the presence of a poly(A) tail. The iNOS 3'-UTR contains AREs (AUUU(U)A $\times 6$ ), which contribute to mRNA stabilization. $(B, C)$ Each construct was introduced into hepatocytes, and the cells were treated with IL-1 $\beta$ (1 $\mathrm{nM})$ in the presence or absence of curcumin $(25 \mu \mathrm{M} / \mathrm{mL})$ for $8 \mathrm{~h}$ for pRiNOS-Luc-SVpA (B) and $5 \mathrm{~h}$ for pRiNOSLuc-3'UTR (C). Luciferase activity was normalized by $\beta$-galactosidase activity. Fold activation was calculated by dividing luciferase activity by control activity (without IL-1 $\beta$ and curcumin). Data are means \pm SD for $n=4$ dishes. $* \mathrm{P}<0.05$ versus IL-1 $\beta$ alone. (D) The cells were treated with IL-1 $\beta(1 \mathrm{nM})$ in the presence or absence of curcumin $(25 \mu \mathrm{M} / \mathrm{mL})$ for the indicated times. Total RNA was analyzed by strand-specific RT-PCR to detect the iNOS gene antisense transcript (AST). 
Curcumin inhibits activation of NF-אB and upregulation of IL-1RI. There are two essential signaling pathways for induction of iNOS, called the I $\mathrm{B}$ kinase and phosphatidylinositol 3-kinase $(\mathrm{PI} 3 \mathrm{~K}) /$ Akt pathways. In the former pathway, IL-1 $\beta$ stimulates degradation of I $\mathrm{B}$ proteins after

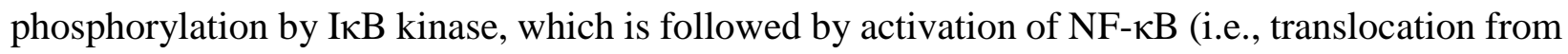
the cytoplasm to the nucleus and DNA binding). Curcumin had no effect on degradation of I $\mathrm{B} \alpha$ after IL-1 $\beta$ stimulation, but rather enhanced its degradation (Fig. 6A). However, an electrophoretic mobility shift assay with nuclear extracts showed that curcumin inhibited NF- $\mathrm{BB}$ activation at 2$5 \mathrm{~h}$, which indicated blockade of NF- $\mathrm{KB}$ nuclear translocation (Fig. 6B).

In the PI3K/Akt pathway, IL-1 $\beta$ stimulates upregulation of IL-1RI by activation of PI3K/Akt [25,26]. Immunoprecipitation-western blotting analysis showed that curcumin inhibited phosphorylation (activation) of Akt, a downstream kinase of PI3K (Fig. 7A). Real-time RT-PCR and western blotting analyses revealed that curcumin reduced expression levels of IL-1RI mRNA and protein (Fig. 7B and 7C).

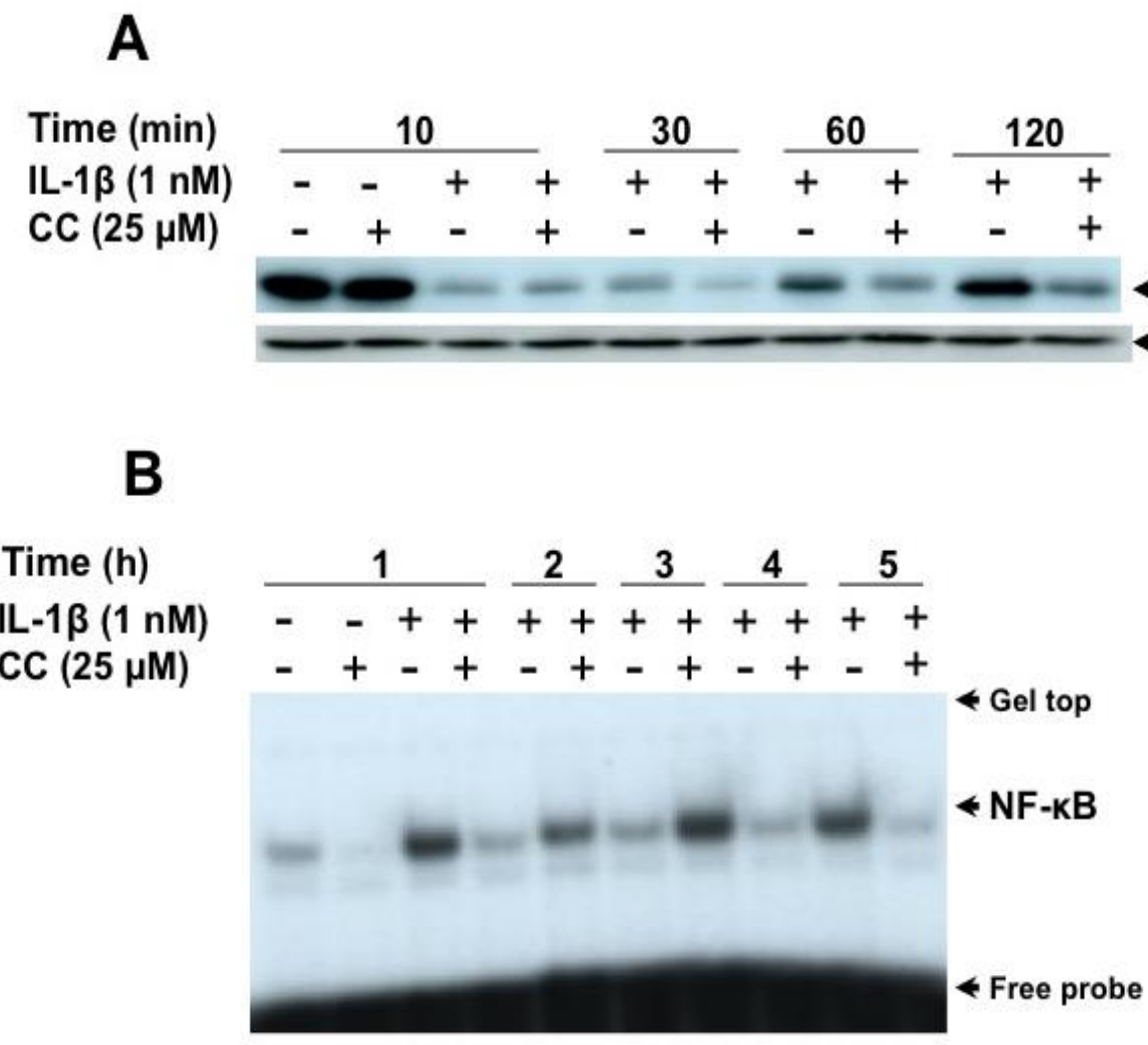

Fig. 6. Effects of curcumin on degradation of $I \kappa B \alpha$ and activation of NF- $\kappa B$. Cells were treated with IL-1 $\beta(1 \mathrm{nM})$ in the presence or absence of curcumin $(25 \mu \mathrm{M})$ for the indicated times. (A) Cell lysates $(20 \mu \mathrm{g}$ of protein) were subjected to SDS-PAGE in a $12.5 \%$ gel, followed by immunoblotting with an anti-IкB $\alpha$ or anti- $\beta$-tubulin antibody. (B) Activation of NF- $\mathrm{BB}$. Nuclear extracts (4 $\mu \mathrm{g}$ of protein) were analyzed by an electrophoretic mobility shift assay. Representative results of three independent experiments are shown. 

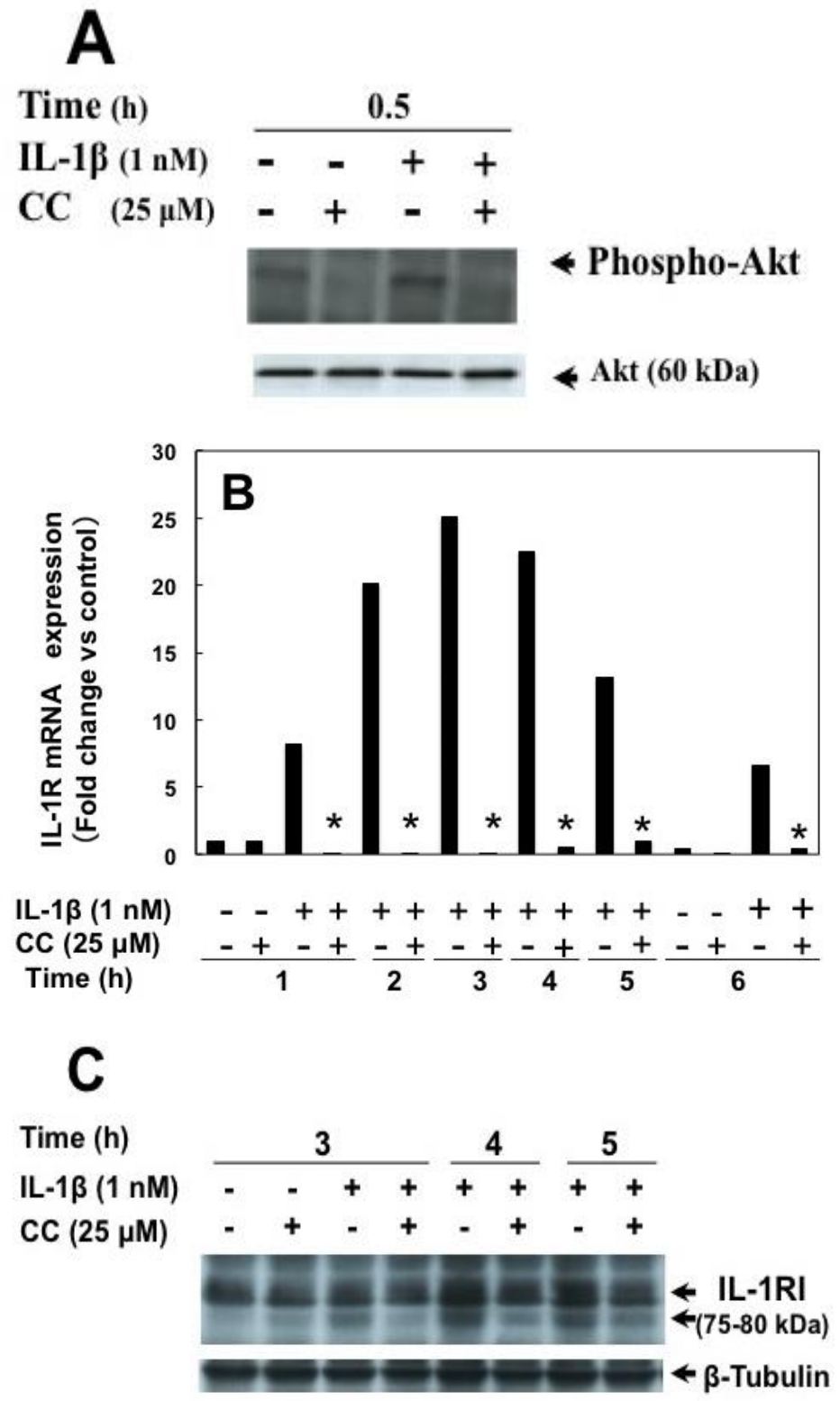

Fig. 7. Effects of curcumin on upregulation of type I IL-1 receptor. Cells were treated with IL-1 $(1 \mathrm{nM})$ in the presence or absence of curcumin $(25 \mu \mathrm{M})$ for the indicated times. (A) Phosphorylation of Akt. Total cell lysates were immunoprecipitated with an anti-Akt antibody, followed by immunoblotting (SDS-PAGE in a gel with a gradient of 6-9\%) with an anti-phospho-Akt or anti-Akt antibody. (B) Total RNA was analyzed by strand-specific RT-PCR to detect type I IL-1 receptor (IL-1RI) mRNA, using EF mRNA as an internal control. (C) Cell lysates (50 $\mu \mathrm{g}$ of protein) were subjected to SDS-PAGE in a $7.5 \%$ gel, and immunoblotted with an anti-IL-1RI or anti- $\beta$-tubulin antibody.

Delayed administration of curcumin inhibits iNOS induction. We examined whether delayed administration of curcumin affects induction of iNOS. Curcumin was added to the medium $0-5 \mathrm{~h}$ after addition of IL-1 $\beta$. Delayed administration of curcumin up to $2 \mathrm{~h}$ after IL-1 $\beta$ addition still markedly inhibited NO production, although the magnitude of inhibition decreased in a timedependent manner (Fig. 8). 


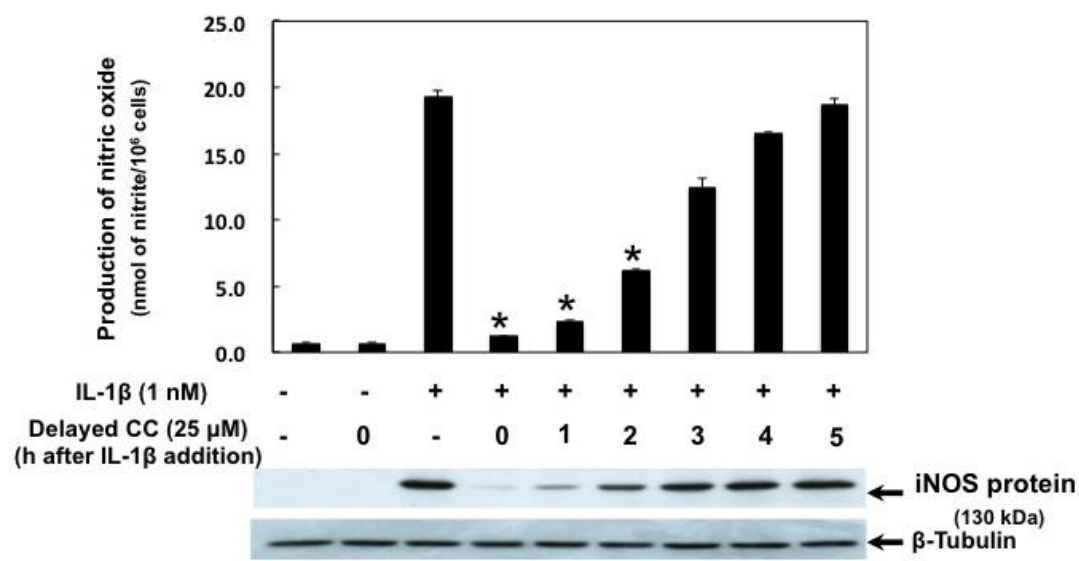

Fig. 8. Effects of delayed glutamic acid administration on induction of iNOS in hepatocytes. Cells were treated with curcumin $(25 \mu \mathrm{M})$ at $0-4 \mathrm{~h}$ after addition of IL-1 $\beta(1 \mathrm{nM})$. The effects of curcumin on NO production (upper panel) and iNOS protein (lower panel) were analyzed at $8 \mathrm{~h}$ after addition of IL-1 $\beta$. Nitrite levels were measured in the culture medium. Data are means $\pm \mathrm{SD}, \mathrm{n}=3$ dishes/point; $* \mathrm{P}<0.05$ versus IL-1 $\beta$ alone. In the western blotting panels, cell lysates (20 $\mu \mathrm{g}$ of protein) were subjected to SDS-PAGE in a $7.5 \%$ gel, and immunoblotted with an antiiNOS or anti- $\beta$-tubulin antibody.

\section{DISCUSSION:}

Curcumin is a pharmacologically safe compound with known anti-inflammatory, anticarcinogenic, and free radical scavenger properties [27-32]. Curcumin supplementation in animal models results in an increase in several cytoprotective enzymes in liver and kidney tissues [33-35]. The antiinflammatory and antioxidant properties of curcumin have been well documented [36-38]. However, the way in which these inhibitory responses are modulated by curcumin is still unclear [39].

In this study, we found that curcumin inhibited induction of iNOS, and even TNF- $\alpha$ and IL-6 in IL-1 $\beta$-stimulated hepatocytes. This finding indicates that curcumin may have anti-inflammatory effects in the liver. Intervention in NF- $\mathrm{B}$ activation may also be beneficial in suppressing toxic/septic shock, graft versus host reactions, radiation damage, and inflammatory reactions. NF$\kappa \mathrm{B}$ plays a major role in inflammation by regulating genes encoding iNOS and proinflammatory cytokines, such as TNF- $\alpha$ [40]. Induction of iNOS gene expression is regulated by iNOS promoter transactivation and by post-transcriptional modifications [24]. Curcumin was recently reported to inhibit NOS [41, 42]. Ex vivo studies have suggested that the inducibility of NOS activity in macrophages is inhibited by $1-20-\mu \mathrm{M}$ concentrations of curcumin [43]. Additionally, administration of an aqueous alkaline solution of curcumin in drinking water significantly inhibits murine hepatic lipopolysaccharide-induced iNOS gene expression [44].

Curcumin also displays anticarcinogenic properties in animals as indicated by its ability to inhibit initiation of tumors when induced by phorbol esters $[33,34]$, which are known to activate 


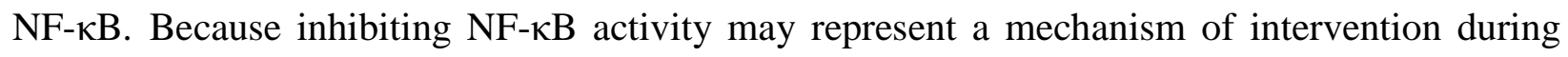
carcinogenesis, curcumin's activity could have considerable implications for chemoprevention of cancer. NF- $\kappa$ B typically exists in the form of p50/65 heterodimers that are attached to its inhibitory

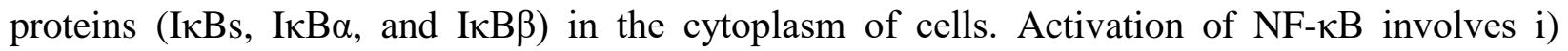
proteolytic degradation of $\mathrm{I} \kappa \mathrm{Bs}$ in the proteosome after phosphorylation by $\mathrm{I} \kappa \mathrm{B}$ kinase, ii) translocation of $\mathrm{NF}-\kappa \mathrm{B}$ to the nucleus, and iii) its binding to the promoter $\kappa \mathrm{B}$ site [45]. In our study, curcumin in primary cultured rat hepatocytes inhibited NF- $\kappa$ B activation (Fig. 6B). We anticipated that curcumin would inhibit $\mathrm{I} \kappa \mathrm{B} \alpha$ degradation, resulting in inhibition of $\mathrm{NF}-\kappa \mathrm{B}$ nuclear translocation as well as in ML-1a (a human myelomonoblastic leukemia cell line) [46]. However, curcumin did not inhibit $\mathrm{I} \kappa \mathrm{B} \alpha$ degradation in this condition (Fig. 6A). Our results indicate that curcumin administration results in inhibition at a step in the signal transduction cascade of NF- $\kappa \mathrm{B}$ activation that occurs after $\mathrm{I} \kappa \mathrm{B}$ phosphorylation, but before the point at which various signals that are transduced by different stimuli converge. Many in vitro studies have reported that curcumin may inhibit NF- $\kappa \mathrm{B}$ and mediate activation of hepatic stellate cells by stimulating peroxisome proliferator-activated receptor- $\gamma$ (PPAR- $\gamma$ ) activity [47]. This leads to inhibition of cellular proliferation and induction of apoptosis of stimulated hepatic stellate cells. Further mechanisms involved in the inhibition of $\mathrm{NF}-\kappa \mathrm{B}$ activation are under investigation.

In concert with $\mathrm{NF}-\kappa \mathrm{B}$ activation, upregulation of IL-1RI by activation of PI3K/Akt is also essential for the induction of iNOS [26]. We found that curcumin decreased IL-1RI mRNA and protein expression (Fig. 7B and 7C) by inhibiting Akt phosphorylation (Fig. 7A). In regards to the activation of Akt, we previously found that inchinkoto [48], hochuekito [49], saireito [50], and genipin [51] inhibited phosphorylation of Akt. These compounds also decreased IL-1RI mRNA and protein expression, similar to curcumin. In contrast, dexamethasone [52], adenosine [53], and glutamic acid [54] inhibit upregulation of IL-1RI, but have no effects on phosphorylation of Akt. Therefore, the mechanisms involved in a reduction of induction of IL-1RI by dexamethasone, adenosine, and glutamic acid are unclear in the present.

Furthermore, in our experiments with iNOS promoter constructs, curcumin was found to inhibit induction of iNOS at the mRNA synthesis and stabilization steps (Fig. 5B and 5C). With regards to iNOS mRNA stabilization, the 3'-UTR of iNOS mRNA in rats has six adenine/uracilrich elements (AREs). These AREs are associated with ARE-binding proteins, such as HuR and the heterogeneous nuclear ribonucleoprotein L/I (PTB), which serve to stabilize mRNA [55]. Recently, we reported that asRNAs are often transcribed from many inducible genes, such as iNOS and TNF- $\alpha$ [56]. The iNOS asRNA interacts with and stabilizes iNOS mRNA [57, 58]. We have reported that drugs, such as FR183998 $\left(\mathrm{Na}^{+} / \mathrm{H}^{+}\right.$exchanger inhibitor $)$[9, 10], insulin-like growth factor-I [11], edaravone (free radical scavenger) [14], inchinkoto [48], hochuekito [49], saireito [50], genipin [51], glutamic acid [54], and sivelestat [59] inhibit iNOS induction. This was 
achieved partly by suppressing iNOS asRNA production in animal models and/or primary cultured hepatocytes. In the current study, curcumin also decreased iNOS asRNA expression (Fig. 5D).

These findings show that curcumin inhibits two essential signaling pathways, NF- $\kappa \mathrm{B}$ activation and IL-1RI upregulation, in the induction of iNOS. Curcumin probably reduces iNOS mRNA expression by inhibition of mRNA synthesis and stabilization, leading to a reduction in iNOS protein and NO production.

Proinflammatory cytokines, such as TNF- $\alpha$, IL- $1 \beta$, and IL-6 play an important role in causing injury to multiple organs, in addition to the liver. Inflammation is a biological response that helps to maintain homeostasis, but excessive inflammation following excessive stress causes organ damage. In our in vitro model, we recently reported that IL-1 $\beta$ stimulated induction of TNF- $\alpha$, parallel with the induction of iNOS [60]. In the current study, curcumin decreased TNF- $\alpha$, CINC1, and IL-6 mRNA expression (Fig. 4A, 4B, and 4C). This finding suggested that curcumin could inhibit induction of inflammatory mediators (iNOS, TNF- $\alpha$, CINC-1, and IL-6), which resulted in liver-protective effects.

In our study, delayed treatment with curcumin after addition of IL-1 $\beta$ caused a significant reduction in NO production and induction of iNOS (Fig. 8). This observation may be of clinical importance because initiation of therapeutic curcumin treatment is usually delayed by the onset of disease. Appropriate regulation of inflammatory reactions during the perioperative period is important in preventing the onset of organ damage and infectious complications, achieving a stable postoperative course and early recovery.

Many studies ranging from basic studies to clinical studies have been conducted on curcumin and its clinical applications in treating various diseases. Based on the range of effects of curcumin, curcumin by itself may be useful in treating and preventing some diseases, but fully establishing its potential will require further studies and clinical applications. Our simple in vitro experiment with cultured hepatocytes may be adequate for the screening of liver-protective drugs because it is rapid and inexpensive compared with in vivo animal models of liver injury.

\section{CONCLUSIONS:}

Curcumin can prevent IL-1 $\beta$-stimulated liver injury in cultured hepatocytes by inhibiting the induction of inflammatory mediators, such as iNOS and TNF- $\alpha$, in part through inhibiting NF- $\kappa \mathrm{B}$ activation. Curcumin may have therapeutic potential for liver injury.

Abbreviations: CC, curcumin; iNOS, inducible nitric oxide synthase; TNF- $\alpha$, tumor necrosis factor-alpha; NO, nitric oxide; IL, interleukin; NF- $\mathrm{B}$, nuclear factor-kappa B; CINC-1, cytokineinduced neutrophil chemoattractant-1; DMSO, dimethyl sulfoxide; LDH, lactate dehydrogenase; SDS-PAGE, sodium dodecyl sulfate-polyacrylamide gel electrophoresis; IL-1RI, the type I interleukin-1 receptor; RT-PCR, reverse transcriptase-polymerase chain reaction. 
Disclosure of potential conflicts of interest: The authors declare that they have no conflicts of interest.

Authors' contributions: RN participated in the design of the study, data collection, statistical analysis, and drafting of the manuscript. TO participated in supervision and provided oversight when the manuscript was being drafted. HH, HM, IM, KM, MK, and MN assisted in the design of the study. MKon provided advice regarding development of the protocol for the study and assisted in the design of the study.

Funding: This work was supported in part by a Grant-in-Aid for Scientific Research from the Ministry of Education, Science, Culture and Sports of Japan, and by grants from the Science Research Promotion Fund of the Japan Private School Promotion Foundation.

\section{REFERENCES:}

1. Kuttan R, Bhanumathy P, Nirmala K, George MC: Potential anticancer activity of turmeric (Curcuma longa). Cancer Lett 1985, 29: 197-202.

2. Chattopadhyay, I., Kaushik, B., Bandyopadhyay, U., Banerjee1, R.K: Turmeric and curcumin: biological actions and medicinal applications. Curr. Sci. 2004, 87: 44-54.

3. Anand P, Kunnumakkara AB, Newman RA, Aggarwal BB: Bioavailability of curcumin: problems and promises. Mol Pharm 2007, 4: 807-818.

4. Aggarwal BB, Surh YJ, Shihsodia S: The molecular targets and therapeutic uses of curcumin in health and disease. Adv Exp Med Biol 2007, 595-598.

5. Kanai M, Imaizumi A, Otsuka Y, Sasaki H, Hashiguchi M, et al.: Dose-escalation and pharmacokinetic study of nanoparticle curcumin, a potential anticancer agent with improved bioavailability, in healthy human volunteers. Cancer Chemother Pharmacol 2012, 69: 65-70.

6. Colasanti M, Suzuki H: The dual personality of NO. Trends Pharmacol Sci 2000, 21: 249-252.

7. Tsuchiya H, Kaibori M, Yanagida H, Yokoigawa N, Kwon AH, et al.: Pirfenidone prevents endotoxin-induced liver injury after partial hepatectomy in rats. J Hepatol 2004, 40: 94-101.

8. Tsuji K, Kwon AH, Yoshida H, Qiu Z, Kaibori M, et al.: Free radical scavenger (edaravone) prevents endotoxin-induced liver injury after partial hepatectomy in rats. J Hepatol 2005, 42: 94-101.

9. Tanaka H, Uchida $\mathrm{Y}$, Kaibori M, Hijikawa T, Ishizaki M, et al.: $\mathrm{Na}+\mathrm{H}+$ exchanger inhibitor, FR183998, has protective effect in lethal acute liver failure and prevents iNOS induction in rats. J Hepatol 2008, 48: 289-299. 
10. Ishizaki M, Kaibori M, Uchida Y, Hijikawa T, Tanaka H, et al.: Protective effect of FR183998, a $\mathrm{Na}+\mathrm{H}+$ exchanger inhibitor, and its inhibition of iNOS induction in hepatic ischemiareperfusion injury in rats. Shock 2008, 30: 311-317.

11. Hijikawa T, Kaibori M, Uchida Y, Yamada M, Matsui K, et al.: Insulin-like growth factor 1 prevents liver injury through the inhibition of TNF-alpha and iNOS induction in Dgalactosamine and LPS-treated rats. Shock 2008, 29: 740-747.

12. Kitade H, Sakitani K, Inoue K, Masu A, Kawada N, et al.: Interleukin-1b markedly stimulates nitric oxide formation in the absence of other cytokines or lipopolysaccharide in primary cultured rat hepatocytes, but not in Kupffer cells. Hepatology 1996, 23: 797-802.

13. Nakanishi H, Kaibori M, Teshima S, Yoshida H, Kwon AH, et al.: Pirfenidone inhibits the induction of iNOS stimulated by interleukin-1beta at a step of NF-kappaB DNA binding in hepatocytes. J Hepatol 2004, 41: 730-736.

14. Yoshida H, Kwon AH, Kaibori M, Tsuji K, Habara K, et al.: Edaravone prevents iNOS expression by inhibiting its promoter transactivation and mRNA stability in cytokine-stimulated hepatocytes. Nitric Oxide 2008, 18: 105-112.

15. Kaibori M, Okumura T, Sato K, Nishizawa M, Kon M: Inducible Nitric Oxide Synthase Expression in Liver Injury: Liver Protective Effects on Primary Rat Hepatocytes. Inflamm Allergy Drug Targets 2015, 14: 77-83.

16. Seglen PO: Preparation of isolated rat liver cells. Methods Cell Biol 1976, 13: 29-83.

17. Horiuti Y, Ogishima M, Yano K, Shibuya Y: Quantification of cell nuclei isolated from hepatocytes by cell lysis with nonionic detergent in citric acid. Cell Struct Funct 1991, 16: 203 207.

18. Green LC, Wagner DA, Glogowski J, Skipper PL, Wishnok JS, et al.: Analysis of nitrate, nitrite, and [15N]nitrate in biological fluids. Anal Biochem 1982, 126: 131-138.

19. Chomczynski P, Sacchi N: Single-step method of RNA isolation by acid guanidinium thiocyanate-phenol-chloroform extraction. Anal Biochem 1987, 162: 156-159.

20. Nishizawa M, Nakajima T, Yasuda K, Kanzaki H, Sasaguri Y, et al.: Close kinship of human 20alpha-hydroxysteroid dehydrogenase gene with three aldo-keto reductase genes. Genes Cells 2000, 5: 111-125.

21. Oda M, Sakitani K, Kaibori M, Inoue T, Kamiyama Y, et al.: Vicinal dithiol-binding agent, phenylarsine oxide, inhibits inducible nitric-oxide synthase gene expression at a step of nuclear factor-kappaB DNA binding in hepatocytes. J Biol Chem 2000, 275: 4369-4373.

22. Bradford MM: A rapid and sensitive method for the quantitation of microgram quantities of protein utilizing the principle of protein-dye binding. Anal Biochem 1976, 72: 248-254.

23. Matsui K, Kawaguchi Y, Ozaki T, Tokuhara K, Tanaka H, et al.: Effect of active hexose correlated compound on the production of nitric oxide in hepatocytes. JPEN J Parenter Enteral Nutr 2007, 31: 373-380; discussion 380-371. 
24. Kleinert H, Pautz A, Linker K, Schwarz PM: Regulation of the expression of inducible nitric oxide synthase. Eur J Pharmacol 2004, 500: 255-266.

25. Yamada M, Nishizawa M, Nakatake R, Habara K, Yoshida H, et al.: Characterization of alternatively spliced isoforms of the type I interleukin-1 receptor on iNOS induction in rat hepatocytes. Nitric Oxide 2007, 17: 98-105.

26. Teshima S, Nakanishi H, Nishizawa M, Kitagawa K, Kaibori M, et al.: Up-regulation of IL-1 receptor through PI3K/Akt is essential for the induction of iNOS gene expression in hepatocytes. J Hepatol 2004, 40: 616-623.

27. Azuine MA, Bhide SV: Chemopreventive effect of turmeric against stomach and skin tumors induced by chemical carcinogens in Swiss mice. Nutr Cancer 1992, 17: 77-83.

28. Conney AH, Lysz T, Ferraro T, Abidi TF, Manchand PS, et al.: Inhibitory effect of curcumin and some related dietary compounds on tumor promotion and arachidonic acid metabolism in mouse skin. Adv Enzyme Regul 1991, 31: 385-396.

29. Huang MT, Smart RC, Wong CQ, Conney AH: Inhibitory effect of curcumin, chlorogenic acid, caffeic acid, and ferulic acid on tumor promotion in mouse skin by 12-O-tetradecanoylphorbol13-acetate. Cancer Res 1988, 48: 5941-5946.

30. Huang MT, Wang ZY, Georgiadis CA, Laskin JD, Conney AH: Inhibitory effects of curcumin on tumor initiation by benzo[a]pyrene and 7,12-dimethylbenz[a]anthracene. Carcinogenesis 1992, 13: 2183-2186.

31. Tanaka T, Makita H, Ohnishi M, Hirose Y, Wang A, et al.: Chemoprevention of 4-nitroquinoline 1-oxide-induced oral carcinogenesis by dietary curcumin and hesperidin: comparison with the protective effect of beta-carotene. Cancer Res 1994, 54: 4653-4659.

32. Ammon HP, Safayhi H, Mack T, Sabierij J: Mechanism of anti- inflammatory actions of curcumin and boswelic acids. J. Ethnopharmacol 1993, 38: 113-119.

33. Sharma RA, Gescher AJ, Steward WP: Curcumin: the story so far. Eur J Cancer 2005, 41: 19551968.

34. Iqbal M, Sharma SD, Okazaki Y, Fujisawa M, Okada S: Dietary supplementation of curcumin enhances antioxidant and phase II metabolizing enzymes in ddY male mice: possible role in protection against chemical carcinogenesis and toxicity. Pharmacol Toxicol 2003, 92: 33-38.

35. Gaedeke J, Noble NA, Border WA: Curcumin blocks multiple sites of the TGF-beta signaling cascade in renal cells. Kidney Int 2004, 66: 112-120.

36. Ammon HP, Wahl MA: Pharmacology of Curcuma longa. Planta Med 1991, 57: 1-7.

37. Satoskar RR, Shah SJ, Shenoy SG: Evaluation of anti-inflammatory property of curcumin (diferuloyl methane) in patients with postoperative inflammation. Int J Clin Pharmacol Ther Toxicol 1986, 24: 651-654. 
38. Shankar TN, Shantha NV, Ramesh HP, Murthy IA, Murthy VS: Toxicity studies on turmeric (Curcuma longa): acute toxicity studies in rats, guineapigs \& monkeys. Indian J Exp Biol 1980, 18: 73-75.

39. Mazidi M, Karimi E, Meydani M, Ghayour-Mobarhan M, Ferns GA: Potential effects of curcumin on peroxisome proliferator-activated receptor- $\gamma$ in vitro and in vivo. World J Methodol 2016, 6: 112-117.

40. Lawrence T: The nuclear factor NF-кB pathway in inflammation. Cold Spring Harb Perspect Biol 2009, 1: a001651.

41. Ohshima $\mathrm{H}$, Bartsch $\mathrm{H}$ : Chronic infections and inflammatory processes as cancer risk factors: possible role of nitric oxide in carcinogenesis. Mutat Res 1994, 305: 253-264.

42. Halliwell B: Free radicals, antioxidants, and human disease: curiosity, cause, or consequence? Lancet 1994, 344: 721-724.

43. Brouet I, Ohshima H: Curcumin, an anti-tumour promoter and anti-inflammatory agent, inhibits induction of nitric oxide synthase in activated macrophages. Biochem Biophys Res Commun 1995, 206: 533-540.

44. Chan MM, Huang HI, Fenton MR, Fong D: In vivo inhibition of nitric oxide synthase gene expression by curcumin, a cancer preventive natural product with anti-inflammatory properties. Biochem Pharmacol 1998, 55: 1955-1962.

45. Akira S, Kishimoto T: NF-IL6 and NF-kappa B in cytokine gene regulation. Adv Immunol 1997, 65: 1-46.

46. Singh S, Aggarwal BB: Activation of transcription factor NF-kappa B is suppressed by curcumin (diferuloylmethane). J Biol Chem 1995, 270: 24995-25000.

47. $\mathrm{Xu} \mathrm{J}, \mathrm{Fu} \mathrm{Y}$, Chen A: Activation of peroxisome proliferator-activated receptor-gamma contributes to the inhibitory effects of curcumin on rat hepatic stellate cell growth. Am J Physiol Gastrointest Liver Physiol 2003, 285: G20-30.

48. Matsuura T, Kaibori M, Araki Y, Matsumiya M, Yamamoto Y, et al.: Japanese herbal medicine, inchinkoto, inhibits inducible nitric oxide synthase induction in interleukin-1 $\beta$-stimulated hepatocytes. Hepatol Res 2012, 42: 76-90.

49. Matsumiya M, Kaibori M, Araki Y, Matsuura T, Oishi M, et al. Japanese herbal medicine hochuekkito inhibits the expression of proinflammatory biomarker, inducible nitric oxide synthase, in hepatocytes. Medicinal Chemistry (Medchem) 2: 112. doi:10.4172/21610444.1000112

50. Miki H, Tokuhara K, Oishi M, Nakatake R, Tanaka Y, et al.: Japanese Kampo Saireito Has a Liver-Protective Effect Through the Inhibition of Inducible Nitric Oxide Synthase Induction in Primary Cultured Rat Hepatocytes. JPEN J Parenter Enteral Nutr 2016, 40: 1033-1041. 
51. Nakatake R, Tsuda T, Matsuura T, Miki H, Hishikawa H, et al.: Genipin inhibits the induction of inducible nitric oxide synthase through the inhibition of NF- $\mathrm{KB}$ activation in rat hepatocytes. Drug Metab Lett. 2016 Oct 20. [Epub ahead of print]

52. Ozaki T, Habara K, Matsui K, Kaibori M, Kwon AH, et al.: Dexamethasone inhibits the induction of iNOS gene expression through destabilization of its mRNA in proinflammatory cytokine-stimulated hepatocytes. Shock 2010, 33: 64-69.

53. Tanaka Y, Ohashi S, Ohtsuki A, Kiyono T, Park EY, et al.: Adenosine, a hepato-protective component in active hexose correlated compound: its identification and iNOS suppression mechanism. Nitric Oxide 2014, 40: 75-86.

54. Nakatake R, Kaibri M, Miki H, Tsuda T,Ueyama Y, et al: Glutamic Acid Has a Liver-Protective Effect through the Suppression of Inducible Nitric Oxide Synthase in Primary Cultured Rat Hepatocytes. IJARCS 2015, 2: 47- 63.

55. Pautz A, Linker K, Hubrich T, Korhonen R, Altenhöfer S, et al.: The polypyrimidine tractbinding protein $(\mathrm{PTB})$ is involved in the post-transcriptional regulation of human inducible nitric oxide synthase expression. J Biol Chem 2006, 281: 32294-32302.

56. Nishizawa M, Ikeya Y, Okumura T, Kimura T. Post-transcriptional inducible gene regulation by natural antisense RNA. Front Biosci. 2015; 20(1): 1-39.

57. Matsui K, Nishizawa M, Ozaki T, Kimura T, Hashimoto I, et al.: Natural antisense transcript stabilizes inducible nitric oxide synthase messenger RNA in rat hepatocytes. Hepatology 2008, 47: 686-697.

58. Yoshigai E, Hara T, Araki Y, Tanaka Y, Oishi M, et al.: Natural antisense transcript-targeted regulation of inducible nitric oxide synthase mRNA levels. Nitric Oxide 2013, 30: 9-16.

59. Araki Y, Matsumiya M, Matsuura T, Kaibori M, Okumura T, et al.: Sivelestat suppresses iNOS gene expression in proinflammatory cytokine-stimulated hepatocytes. Dig Dis Sci 2011, 56: 1672-1681.

60. Yoshigai $\mathrm{E}$, Hara $\mathrm{T}$, Inaba $\mathrm{H}$, Hashimoto I, Tanaka $\mathrm{Y}$, et al.: Interleukin-1 $\beta$ induces tumor necrosis factor- $\alpha$ secretion from rat hepatocytes. Hepatol Res 2014, 44: 571-583. 